\title{
Does India's Menstrual Hygiene Management Scheme exclude the disabled?
}

\section{DAISY DUTTA, CHHANDA CHAKRABORTI}

\begin{abstract}
The Menstrual Hygiene Scheme of the Government of India wishes to generate awareness among adolescent girls, and provide them with hygienic and affordable sanitary napkins. The scheme has been criticised for many reasons by various reviewers. However, we draw attention to a hitherto unaddressed gap that the scheme has effectively overlooked, the menstrual hygiene needs of disabled adolescent girls, and has thereby denied them their right to health and healthcare. This exacerbates health disparities, and raises questions of public health ethics. We conclude with recommendations on how to redress the situation and make the scheme more inclusive.
\end{abstract}

Keywords: Menstrual hygiene management, India, adolescent girls, disability, right to health, health disparities, public health ethics.

\section{Introduction}

Menstruation is a natural biological process. Menstrual hygiene management (MHM) is the management of menstruation through the use of hygienic absorbents, following personal hygiene during menstruation, and safe and proper disposal of the absorbents. This is crucial for the health and well-being of women and girls, for poor MHM is known to lead to health problems such as toxic shock syndrome, reproductive tract infections, and urinary tract infections (1-3).

Data from the National Family Health Survey 2015-16 (NFHS4) ${ }^{1}$ show that overall, only $57.6 \%$ of Indian women in the age group of 15-24 use a hygienic method (sanitary napkins or freshly made new cloth pads) for menstrual protection (4).

Author: Daisy Dutta (corresponding author - daisydutta@iitkgp.ac.in), Senior Research Fellow, Department of Humanities \& Social Science, Indian Institute of Technology, Kharagpur, West Bengal, 721302 INDIA; Chhanda Chakraborti (cchanda@hss.iitkgp.ac.in), Professor, Department of Humanities and Social Sciences, Indian Institute of Technology Kharagpur, West Bengal 721302, INDIA.

To cite: Dutta D. Chakraborti C. Does India's Menstrual Hygiene Management Scheme exclude the disabled?. Indian J Med Ethics. Published online first on October 26, 2021. DOI: 10.20529/IJME.2021.081

Manuscript Editor: Sandhya Srinivasan

Peer reviewer: Lakshmi Lingam

c Indian Journal of Medical Ethics 2021
Lack of adequate awareness about menstruation and MHM, lack of WASH (water, sanitation and hygiene) facilities, lack of social support, lack of accessibility, availability and affordability of hygienic menstrual management products, and of their easy and safe disposal, have been noted as some of the main and usual barriers which women and girls, in general, face for the practice of adequate MHM in India (5).

The Government of India's (GOI) Menstrual Hygiene Scheme (MHS) (6), started in 2011, is directed primarily at girls from 1019 years in rural India, with the twin objectives of increasing awareness about menstrual hygiene, and increasing access to affordable and hygienic menstrual products. The scheme is run through accredited social health activists (ASHAs), community-based groups, and school teachers.

This paper presents a critique of MHS, but with a difference. Earlier review of the scheme has identified various lacunae in its implementation, such as, irregular supply of subsidised sanitary napkins, poor quality of napkins, failure of ASHAs (7). However, this paper does not wish to discuss these implementation shortfalls of MHS; since these are relatively easily remediable through better supply chain management, and better-quality management.

Instead, in this paper, we draw attention to a more fundamental, and what, to the best of our belief, is a hitherto uncited limitation of this scheme; namely, its complete oversight of the menstrual health needs of adolescent girls with disabilities. We argue for the acknowledgement of the special menstrual needs of adolescent girls with disabilities in the union government's MHS.

\section{Understanding disability}

According to a definition from the World Health Organization (WHO) (8), disability as a term refers not just to the impairments, but also to the limitations, and barriers that may exist between the disabled and their living environment; and which hinder their full and effective participation on an equal basis in society with the able-bodied population. The WHO definition implies that disability should be understood along with the responsibility of a fair and inclusive society to remove these barriers for the disabled for their better participation as members of society. The Rights of Persons with Disabilities Act 2016, India, also seems to reiterate the same point when it states that a person with disability is "a person with long term physical, mental, intellectual or sensory impairment which, in 
interaction with barriers, hinders his full and effective participation in society equally with others." (9). The Act too recommends enabling measures for the disabled to improve their social participation. In this paper, we follow this understanding of disability, which refers not only to impairments, but also to the barriers that the disabled face and to the duty to remove those barriers as far as possible.

As per Census 2011, India has 11.8 million females living with disability (10). Out of which, 2.05 million fall in the age group of 10-19 years, with one or the other of the 21 specified disabilities mentioned in The Rights of Persons with Disabilities Act, 2016. When MHS targets the adolescent girls of India, this group is also supposed to be part of the target population. We argue that the MHS shows no acknowledgement of the menstrual needs of this group in either its planning or its implementation.

\section{Challenges adolescent girls with disabilities face in MHM}

As already mentioned, practice of safe MHM is not barrierfree for able-bodied girls and women. From this, we may infer the level and layers of difficulties that girls and women with disabilities may face for safe MHM.

Disabilities may be broadly divided into two categories: intellectual and physical. A systematic review of menstrual hygiene management requirements for the disabled found that adolescent girls with intellectual impairments often experience difficulties in managing their menstruation. This makes it difficult to use behavioural change therapies to eliminate "inappropriate actions" in relation to menstrual blood and menstrual absorbents. For example, the prevalence of pre-menstrual syndrome or PMS among girls with autism was found to be significantly higher, and this higher prevalence of PMS was found to have adverse effects on their MHM through behavioural change $(11,12)$.

Adolescent girls with intellectual disabilities often require more counselling and guidance for menstrual hygiene management. Also, since girls with severe intellectual disability often face difficulty in communicating and in understanding instructions provided, their caregivers must be trained to anticipate their menstrual needs and to understand their uncommunicated needs. Studies indicate that adolescent girls with intellectual impairment may refuse to wear menstrual absorbents, and may on occasion change their menstrual absorbent in front of other people (13). This shows that their safe MHM depends a lot on the communication and the training of their caregivers.

On the other hand, physical disability imposes a different set of barriers for MHM practice, in terms of access and effective utilisation of the water, sanitation and hygiene infrastructure and facilities. For example, women and girls with spinal cord injuries and paraplegia cannot be expected to use standard toilets for changing their menstrual absorbents. Girls with cerebral palsy may require help while changing their menstrual absorbents and other hygienic menstrual practices (14). In the absence of caregivers, they may be obliged to crawl on the floor to use the toilet, exposing themselves to more infections (13). Some women and girls with physical impairments may be more vulnerable to vaginal infections. A study of adolescents with cerebral palsy reported that $58.3 \%$ of adolescents reported some form of vaginal infection; $71.4 \%$ reported white vaginal discharge (14). Visually impaired girls may have difficulties positioning their menstrual absorbents, or gauging the proper absorbency of the menstrual blood, unless they have an attendant caretaker. They may not be able to identify by themselves the onset and end of their menstruation, or the intensity of their menstrual flow and leakages.

Caregivers and the medical profession have sometimes responded to these MHM challenges for physically and intellectually impaired girls and women with ethically questionable practices. There have been reports of girls with disabilities being subjected to forced sterilisation, including hysterectomies $(15,16)$. Girls with intellectual difficulties may be subjected to these procedures as matters of convenience to eliminate the need to manage menstruation, and to prevent pregnancy following sexual abuse (15), which does not address the need to prevent sexual abuse. Such instances are more common among those with severe intellectual disability (16). In a society which does not value girl children, the caregivers of girls with disabilities may not always give them the substantial support that is needed.

This is all the more reason that the MHS should recognise the special menstrual health needs of girls with disabilities, and should include interventions that enable girls and women with disability to manage their own menstrual hygiene to the extent possible, and with as much dignity as possible. Such interventions addressing the needs of the most disadvantaged are an ethical requirement of any public health programme.

\section{How the MHS fails disabled girls}

Unfortunately, deep-seated patriarchy and gender bias are entrenched in almost every stratum of Indian society. A society which considers its girl children as liabilities is expected not to be sensitive to the needs of its disabled girls. This is reflected also in the planning and implementation of the MHS.

Certain details describing how the scheme is implemented indicate that they were designed keeping in mind the MHM needs of typical adolescent girls, and no provisions have been made to reach girls with disabilities.

First, MHS aims to distribute affordable sanitary napkins at a subsidised price by the ASHAs or SHGs, and at schools through the School Health Promotion or nodal school teachers. However, the scheme does not describe any provision to provide these products to girls with physical or intellectual disabilities. These girls may not attend schools for 
general students, and they may not be able to go to the health centres where the ASHAs are located. The ASHAs, on the other hand, are assigned many other duties, the workload of which obstructs them from conducting monthly meetings for MHM. Expecting the overloaded ASHAs to go door-todoor for distribution of sanitary napkins for the special-needs group may not be viable. So, in the final analysis, disabled girls fall into the most disadvantaged group, in terms of their menstrual health status. They are likely to depend on a sympathetic person or a caregiver, bringing the napkins to them. Either way, the MHS, if it aspires to be a fair distribution arrangement, should arrange for products to reach the most disadvantaged, when the needy cannot reach the products.

Second, the MHM uses conventional classroom teaching and discussion methods with a chalk board and diagrams for MHM awareness generation programmes. Such a pedagogical style and method may suit typical school-going girls; but not an adolescent girl with impairment. For example, the visually challenged will need a Braille instrument; a hearing-impaired girl may require sign language facilities. Similarly, a girl with intellectual disabilities may need a different level of counselling or training. However, MHS works with a blanket principle of homogeneity. It has no provision for catering to special or individual needs. For example, it has no special arrangement or mechanism for disseminating MHM-related information for the disabled in general. It has no room for special counselling for the impaired, or special training for caregivers, to respond to the special menstrual health needs of adolescent girls with different kinds of impairments.

The need for trained caregivers is more acute in cases of MHM for the disabled $(12,13,14)$. However, there is no mention in the MHS to sensitise or provide special training to caregivers of disabled girls.

And, finally, ASHAs are required to play a crucial role in the MHS. However, as mentioned, the Ministry of Health and Family Welfare (MoHFW, Gol) has assigned far too many responsibilities to ASHAs, who are, therefore often unable to do all that is required of them.

\section{The MHS violates policy and law}

The design of the MHS is contrary to the spirit of the National Policy for Persons with Disabilities (2006). This policy acknowledges persons with disability as a valuable human resource for the country, and seeks to create an environment that provides them with equal opportunities, protection of their rights, and full participation in society (10).

The Rights of Persons with Disabilities Act, 2016, recognises the rights of the disabled to "full and effective participation and inclusion in society". The government is expected to "ensure that the persons with disabilities enjoy the right to equality, life with dignity and respect for his or her integrity equally with others". Specifically, the Act requires the government to "take steps to utilize the capacity of persons with disabilities by providing appropriate environment" (9). This statement implies that before providing equal opportunities to disabled persons, the government is duty bound to take the steps necessary for them to avail of these equal opportunities.

The MHS fails to honour these rights, as it has failed to create an appropriate environment for disabled adolescents to facilitate their access to the scheme's benefits. Any initiative to promote menstrual hygiene management in the country must be inclusive. The needs of adolescent girls with disabilities remain unfairly overlooked in the scheme as it is currently constructed.

\section{Right to health and healthcare}

In India, though the Right to Health has not so far been recognised as a human right, it is considered a part of a fundamental right flowing from Article 21 of the Constitution. Article 21 incorporates the provisions for the right to life and personal liberty to everyone. All citizens have the right to proper healthcare and protection from disease and health hazards. The government also has an obligation to provide healthcare facilities to everyone.

This fundamental right is compromised when a national health scheme, such as MHS, overlooks the health needs and health hazards faced by a segment of the population that is already disadvantaged. The right to health is also a fundamental right of persons with disabilities, who have the right to all community support services and they must not be discriminated against on the basis of their disabilities (9).

The MHS appears to be discriminatory in its oversight of this population. By the oversight, it neglects the right to health to adolescent girls with disabilities, and thus violates their constitutional and fundamental rights.

This failure to consider the needs of disabled adolescent girls in a programme meant specifically to address the vulnerability of adolescent girls illustrates the routine neglect of the disabled in health policies and programmes. The invisibility of the disabled is more glaring in the disabled adolescent girl who is presumed to have no sexual and reproductive health needs. This is contrary to the ethics of public health programmes.

Disparities in health status among different groups of the population often show up in terms of various social determinants, such as age, gender, caste, and affect the disadvantaged groups (based on race, religion, nationality, gender, and disability) in a society most severely.

Health disparities due to unhygienic management of menstruation among adolescent girls with disabilities are potent enough to lead to inequalities in health outcomes (17). Adolescent girls with disabilities are already more vulnerable. Their exclusion from a national health scheme can only increase this vulnerability. The remedy lies in a more equitable distribution of the benefits from MHS services. 


\section{Recommendations}

The database of disabled persons in India (10) could have helped the MHS to consider and include the MHM needs of adolescent girls with disabilities. The scheme could have included mechanisms to ensure that all adolescent girls had access to facilities such as menstrual absorbents, and awareness programmes. Even now, it is not too late to revise the scheme and to expand the circle of its reach.

An amended disability-friendly menstrual hygiene scheme should also consider the following issues: engagement, universal teaching designs, barrier-free technologies, and sensitising and training caregivers and providers.

The MHS must engage with persons with disabilities, as well as with experts, to address the challenges that disabled girls and women face when managing their menstrual hygiene. The involvement of non-governmental organisations can also reduce these barriers.

The MHS should follow the practice of "universal design" or "the design of the products, environments, programs and services to be usable by all people to the greatest extent possible, without the need for adaptation or specialized design and shall apply to assistive devices including advanced technologies for particular group of persons with disabilities" (9). For example, instead of relying on the conventional visual and auditory means of communication for awareness programmes, one may consider consistent inclusion of universal designs of communication, such as sign language and Braille. Awareness programmes about MHM should be relevant and comprehensible to all.

The MHS must include in its objectives, facilities such as the provision of barrier-free toilets; the manufacture and provision of menstrual hygiene products made with special consideration for the ease of use by the differently-abled; ensuring ease of access to menstrual waste disposal facilities.

The role of well-trained healthcare providers is also crucial to help girls with disabilities to address the challenges faced in an adequate manner. The MHS should impart special sensitisation and training on $\mathrm{MHM}$ to caregivers to the differently-abled.

\section{Conclusion}

To avoid inequity and growing health disparity, and social exclusion, in a well-intended scheme, the MHS needs to open itself to the special menstrual health needs of adolescent girls with disabilities. It needs to change its vision to be more inclusive in its approach, design and methods. There should be equitable access to MHM-related information and healthcare services for all adolescent girls, the non-disabled as well as those with disabilities.

\section{Competing interest and funding: None to declare.}

${ }^{1}$ Note: The most recent NFHS-5 has not released the overall national data yet; it has only released the key findings of some states. Therefore, we cannot include NFHS-5 data for all Indian women who use hygienic methods of menstrual protection.

\section{References}

1. Das P, Baker KK, Dutta A, Swain T, Sahoo S, Das BS, et al. Menstrual hygiene practices, WASH access and the risk of urogenital infection in women from Odisha, India. PLoS One. 2015 Jun 30;10(6):e0130777.

2. Kreem HEA, Ayed MMA, Mostafaa NEH. Assessment and promotion awareness level and practice about menstruation of rural preparatory school girls at Assuit City, Egypt. IOSR Journal of Nursing and Health Science. 2016 May-Jun; 5 (3): 12-21

3. Tiwari A, Ekka I J, Thakur R. Assessment of knowledge and practices regarding menstrual hygiene among adolescent girls of Government higher secondary school, station Murhipar, Rajnandgaon (C.G.). International Journal of Community Medicine and Public Health 2018;5(4):1335-8.

4. National Family Health Survey-4: India report. 2015-16. International Institute for Population Sciences, Ministry of Health and Family Welfare, Gol; 2017 Dec.

5. Sommer M. Caruso BA, Sahin M, Calderon T, Cahill S, Mahon T, et al. A time for global action: Addressing girls' menstrual hygiene management needs in schools. PLoS Med. 2016; 13 (2): e1001962.

6. Revised Guideline for Menstrual Hygiene Scheme. 2016. Ministry of Health and Family Welfare, Government of India. 2016 Mar 4[cited 2021 Oct20]. Available from: https://nhm.gov.in/WriteReadData/ I892s/61090433691481276612.pdf

7. Ministry of Human Resource Development, Gol. Performance Audit of Tribal Sub-Plan, Report of the Comptroller and Auditor General. Report No. 33 of 2015. New Delhi: Ministry of Health and Family Welfare and Ministry of AYUSH, Gol. 2015 Dec 8.

8. World Health Organization World Report on Disability. Geneva:WHO; 2011 [cited 2019 April 4]. Available from: https://www.who.int/ disabilities/world_report/2011/report.pdf

9. Ministry of Law and Justice, Gol. The Rights of Persons with Disabilities Act. 2016. New Delhi: MoLJ; 2016 Dec..

10. Ministry of Statistics and Programme Implementation, Gol. Disabled Persons in India: A Statistical Profile. Social Statistics Division, MoSPI; Gol; 2016.

11. Obaydi $H$, Puri BK. Prevalence of premenstrual syndrome in autism: $A$ prospective observer-rated study. J Int Med Res. 2008 Mar-Apr; 36(2): 268-72.

12. Wilbur J, Torondel B, Hameed S, Mahon T, Kuper H. Systematic review of menstrual hygiene management requirements, its barriers and strategies for disabled people. PLoS One. 2019 Feb 6;14 (2): e0210974.

13. House $S$, Mahon T, Cavill S; co-published by WaterAid and 17 other organizations. Menstrual Hygiene Matters; A resource for improving menstrual hygiene around the world. Reprod Health Matters. 2012;21(41):257-9.

14. Rao A P, Shah H, Guruvare S, Guddattu V. Growth, sexual development and menstrual issues among girls with cerebral palsy - A cross sectional study in a tertiary care centre. Clin Epidemiology Glob Health. 2019 Sep; 7(3): 367-71. https://doi.org/10.1016/j.cegh. 2018.08.003

15. Patage DP, Sharankumar H, Bhimayya B, Ayesha N, Kiran S. Reproductive and sexual health needs among differently-abled individuals in the rural field practice area of a medical college in Karnataka, India. Int J Med Sci Public Health. 2016;4 (7): 964-8.

16. Marquez-Gonzalez H, Valdez-Martinez E, Bedolla M. 2018. Hysterectomy for the management of menstrual hygiene in women with intellectual disability. A systematic review focusing on standards and ethical considerations for developing countries. Front Public Health. 2018; 6 (338): 1-18.

17. Braveman PA, Kumanyika S, Fielding J, Laveist T, Borell et al. 2011. Health disparities and health equity: The issue is justice. Am J Public Health. 2011 Dec;101 (S1):S149-S155. 